\title{
New Perspectives on $\beta$-Adrenergic Mediation of Innate and Learned Fear Responses to Predator Odor
}

\author{
Fabrício H. M. Do Monte, ${ }^{1}$ Newton Sabino Canteras, ${ }^{2}$ Daniel Fernandes, ${ }^{1}$ Jamil Assreuy, ${ }^{1}$ and Antonio P. Carobrez ${ }^{1}$ \\ ${ }^{1}$ Departamento de Farmacologia, Centro de Ciências Biológicas, Universidade Federal de Santa Catarina, Florianópolis-SC 88040900, Brazil, and \\ ${ }^{2}$ Departamento de Anatomia, Instituto de Ciências Biomédicas, Universidade de São Paulo, São Paulo-SP 05508900, Brazil
}

\begin{abstract}
In the present study, we investigated the role of noradrenergic transmission in unconditioned and conditioned responses to predatory threats. First, we examined the effects of systemically injected $\beta$-blockers on unconditioned and contextual conditioned response to cat odor. The centrally acting $\beta$-blocker (propranolol) was able to impair unconditioned responses, as well as the acquisition of the contextual fear to cat odor; however, the peripherally acting (nadolol) was not effective. Next, we examined the neural substrate underlying the noradrenergic modulation of the defensive response to cat odor and focused on the dorsal premammillary nucleus (PMd), because it represents the hypothalamic site most responsive to predatory threats and, at the same time, presents a dense plexus of noradrenergic fibers. We were able to see that propranolol significantly reduced PMd-Fos expression in response to cat odor and that $\beta$-adrenoceptor blockade in the PMd, before cat odor exposure, reduced defensive responses to the cat odor and to the cat odor-related environment. We have also shown that $\beta$-adrenoceptor blockade in the PMd, before the exposure to cat odor-related context, impaired the contextual conditioned responses. Overall, the present results provide convincing evidence suggesting that central noradrenergic mediation is critical for the expression of unconditioned and contextual conditioned antipredatory responses. We have further shown that the PMd appears to be an important locus to mediate these $\beta$-adrenoceptor effects.
\end{abstract}

Key words: noradrenergic system; $\beta$ adrenoceptors; $\beta$-blockers; cat odor; defensive behavior; contextual fear

\section{Introduction}

Noradrenergic transmission has long been implicated in stressrelated changes in behavior (Bremner et al., 1996; Morilak et al., 2005). $\beta$-blocker agents are widely used in anxiety disorders such as social phobia (Brunello et al., 2000; Davidson, 2006), posttraumatic stress disorder (Kent et al., 2002; Vaiva et al., 2003; Giles, 2005), and panic disorder (Sullivan et al., 1999; Hirschmann et al., 2000). In animal models of anxiety, the $\beta$-blocker propranolol was able to promote anxiolytic effects observed in the elevated plus maze (Audi et al., 1991; Gorman and Dunn, 1993; Stern et al., 2008) and in the open field (Angrini et al., 1998). Moreover, evidence from many different types of experiments indicates that the adrenomedullary hormone epinephrine (adrenaline), released during and after emotionally arousing experiences, plays a critical role in consolidating lasting memories (for review, see McGaugh, 2004).

Despite the importance of the adrenomedullary hormones in stress responses and emotionally arousing memory consolidation, there is no data in the literature examining the putative roles

\footnotetext{
Received June 19, 2008; revised July 29, 2008; accepted 0ct. 22, 2008.

This work was supported by Coordenação de Aperfeiçoamento de Pessoal de Nível Superior, Fundação de Apoio à Pesquisa Científica e Tecnológica do Estado de Santa Catarina, Fundação de Amparo à Pesquisa do Estado de São Paulo Grant 05/59286-4, and Conselho Nacional de Desenvolvimento Científico e Tecnológico, from which F.H.M.D. received a master fellowship, D.F a doctoral fellowship, and A.P.C, N.S.C., and J.A. a research fellowship.

Correspondence should be addressed to Antonio P. Carobrez, Departamento de Farmacologia, Universidade Federal de Santa Catarina, Campus Trindade, Florianópolis-SC 88040900, Brazil. E-mail: adepadua@farmaco.ufsc.br.

D0I:10.1523/JNEUROSCI.2843-08.2008

Copyright $\odot 2008$ Society for Neuroscience $\quad 0270-6474 / 08 / 2813296-07 \$ 15.00 / 0$
}

of noradrenergic transmission in unconditioned and conditioned responses to natural threats, such as a predator or its odor. Predatory odors are specific chemical cues incorporated by prey species as phyletic memory or "memory of the species" (Fuster, 1997), providing the recognition, the avoidance, and the expression of defensive behavior against predators (Apfelbach et al., 2005).

In the present investigation, we compared the effects of a centrally acting $\beta$-blocker (propranolol) with a $\beta$-blocker displaying only peripheral action (nadolol) on the unconditioned and contextual conditioned responses to cat odor. Next, we examined a possible neural substrate underlying the noradrenergic modulation of the defensive response to cat odor. Several studies have documented that the hypothalamus plays a major role in processing actual and contextual predatory threats and that the dorsal premammillary nucleus (PMd) represents the hypothalamic site most responsive to predatory threats (Canteras et al., 1997, 2008; Dielenberg et al., 2001; Cezario et al., 2008; Staples et al., 2008). Interestingly, the PMd presents a moderately dense plexus of noradrenergic fibers (Moore and Card, 1984) and, therefore, is likely to be involved in the noradrenergic modulation of antipredatory defensive behavior. We started investigating this possibility by examining the effects of a centrally and a noncentrally acting $\beta$-blocker on the intensity of PMd-Fos immunoreactivity in response to cat odor. Next, we examined how local injections of $\beta$-blocker in the PMd, during exposure to cat odor, interfere with the unconditioned and contextual conditioned responses. In addition, we also examined how $\beta$-blocker applied in the PMd, 
during exposure to cat odor-associated context, interferes with the expression of conditioned contextual responses. Overall, the present results provide convincing evidence suggesting that central noradrenergic mediation is critical for the expression of unconditioned and contextual conditioned antipredatory responses and acts via a specific hypothalamic circuit.

\section{Materials and Methods \\ Subjects}

Male Wistar rats weighing 320-370 g and aged 14-16 weeks at the time of test were used. The animals were housed in groups of three to four per cage $(50 \times 30 \times 15 \mathrm{~cm})$ with standard environmental conditions $(24 \pm$ $1^{\circ} \mathrm{C}$ and a $12 \mathrm{~h} \mathrm{light/dark} \mathrm{cycle,} \mathrm{lights} \mathrm{on} \mathrm{7:00} \mathrm{A.M.)} \mathrm{and} \mathrm{food} \mathrm{and} \mathrm{water}$ available ad libitum. Experiments were performed during the mid-light phase of the light/dark cycle.

\section{Drugs}

Propranolol hydrochloride or nadolol (Sigma-Aldrich) were dissolved $(10 \mathrm{mg} / \mathrm{ml})$ in saline $(0.9 \% \mathrm{NaCl})$, which alone served as a vehicle control, were and administered intraperitoneally in an injection volume of 1 $\mathrm{ml} / \mathrm{kg}$. The dose of $10 \mathrm{mg} / \mathrm{kg}$ intraperitoneally was selected on the basis of previous behavioral pilot studies performed in our laboratory, and its antagonistic efficacy in $\beta$-adrenoceptors was shown in a cardiovascular assay performed in this study. Xilazine $(2.5 \mathrm{mg} / \mathrm{kg}$, Rompun; Bayer) and ketamine ( $15 \mathrm{mg} / \mathrm{kg}$, Dopalen; Agribands) delivered intramuscularly as anesthetic, $30 \mathrm{IU}$ of heparin [diluted in a saline sterile buffered solution with the following (in $\mathrm{mM}$ ): $137 \mathrm{NaCl}, 2.7 \mathrm{KCl}, 1.5 \mathrm{KH}_{2} \mathrm{PO}_{4}$, and 8.1 $\left.\mathrm{NaHPO}_{4}, \mathrm{pH} 7.4\right]$ as anticoagulant, and dopamine $(18,36$, and $72 \mu \mathrm{g} / \mathrm{kg}$, Revivan; Zambon Labs) were used for the cardiovascular assays. Atenolol [(RS)-Atenolol; Tocris Cookson] was dissolved in PBS, which alone served as a vehicle control. Each animal received a bilateral injection of 10 or $40 \mathrm{nmol}$ atenolol or PBS in a volume of $0.2 \mu \mathrm{l}$.

\section{Heart rate measurement}

Rats were anesthetized with xilazine and ketamine, and 30 IU of heparin was administered through a needle inserted in the left femoral vein to prevent clot formation. A polyethylene catheter (PE-10), previously implanted into the right carotid artery, was connected to a pressure transducer coupled to acquisition hardware and software (PowerLab 8/30 running Chart 5.4; AD Instruments), enabling the heart rate measurement and analysis.

\section{Cat odor apparatus}

Testing occurred in a wooden rectangular arena $(60 \times 26 \times 36 \mathrm{~cm})$ comprising two different areas: a covered enclosed box $(20 \times 26 \times 36$ $\mathrm{cm})$ termed "hide box," and an uncovered open area $(40 \times 26 \times 36 \mathrm{~cm})$ according to the apparatus used by Dielenberg et al. (1999). On the front wall of the hide box, a small $(6 \times 6 \mathrm{~cm})$ square hole allows the free transit of the rat between the two areas. On the front wall of the open area, a neutral folded cloth $(45 \times 30 \mathrm{~cm})$ was anchored during familiarization and context sessions, or a similar folded cloth impregnated with a blend of cat fur odor (obtained by rubbing it against the back fur of a domestic cat for $5 \mathrm{~min}$ ) was anchored during the conditioning session. The cat odor cloth was kept in a sealed plastic bag and was used in the experiments on the same day. Subjects were placed in the apparatus facing the cloth in all phases of the experiments. All sessions lasted $10 \mathrm{~min}$. After each trial, the apparatus was cleaned with ethanol solution $(10 \% \mathrm{v} / \mathrm{v})$. The experiments were performed in a low illumination (4 lux) condition room. A video camera was placed $100 \mathrm{~cm}$ above the apparatus, and a recording system was located in an adjacent room. The recording system enabled a trained observer to score, within each session, the following parameters: (1) percentage of approach time: the amount of time the rats are located within a $7 \mathrm{~cm}$ perimeter near the cloth; (2) percentage of hide time: the amount of time the rats spent in the hide box; (3) head-out: the time the rats peep out toward the open area while in the hide box.

\section{Surgery}

Rats $(n=57)$ were anesthetized with xilazine $(2.5 \mathrm{mg} / \mathrm{kg}$, Rompun; Bayer) and ketamine (15 mg/kg, Dopalen; Agribands) and positioned in a stereotaxic frame. Xylocaine $(0.1 \mathrm{ml} ; 2 \mathrm{mg} / \mathrm{ml})$ was subcutaneously injected into the scalp, and a longitudinal incision was made. The bone was then exposed, and two stainless steel guide cannulas (diameter, 0.7 $\mathrm{mm}$; length, $15 \mathrm{~mm}$ ) were stereotaxically implanted with the cannula tips $2.0 \mathrm{~mm}$ above the PMd [coordinates: anteroposterior, $-4.16 \mathrm{~mm}$ from bregma; laterolateral, $\pm 0.6 \mathrm{~mm}$ from midline; dorsoventral, $-6.9 \mathrm{~mm}$ from skull surface (Paxinos and Watson, 1997)]. The cannulas were anchored to the skull with dental cement and two stainless screws previously plugged with a screwdriver. After surgery, rats were allowed to recover 1 week before the cat odor experiments.

\section{Intracerebral injection}

Atenolol or PBS microinjections were administered 10 min before conditioning or context phases by inserting two 17.0-mm-long stainless steel needles through the guide cannula, while the rat was gently restrained. The needles were connected to a $5 \mu \mathrm{l}$ Hamilton microsyringe with polyethylene (PE-10) tubing. An infusion machine (B12000 model; Insight) allowed the bilateral microinjections over a $20 \mathrm{~s}$ time period $(0.6 \mu \mathrm{l} / \mathrm{min})$. The needles were kept within the cannulas for an additional $20 \mathrm{~s}$ after drug infusion to maximize the diffusion and to prevent backflow of the drug into the cannulas. Drug solutions were freshly prepared before each experiment.

\section{Experimental procedures}

Experiment 1: effects of propranolol and nadolol on the heart rate increase produced by dopamine systemic injection. Animals were anesthetized and received intraperitoneal injections of either propranolol $(n=3)$ or nado$\mathrm{lol}(n=3)$ at doses of $10 \mathrm{mg} / \mathrm{kg}$. Thirty minutes later, dopamine $(18,36$, and $72 \mu \mathrm{g} / \mathrm{kg}$ ) was administered in the left femoral vein. Antagonistic effects of both $\beta$-blockers on heart rate increase after dopamine injections were registered for additional analysis.

Experiment 2: effects of propranolol and nadolol on rats' defensive behavior elicited in a cat odor apparatus. The subjects were randomly assigned to one of the three experimental groups [ $(1)$ saline $(n=27),(2)$ propranolol (10 mg/kg, i.p.; $n=18)$, or (3) nadolol (10 mg/kg, i.p.; $n=18)]$ and placed in the apparatus for 3 consecutive days. In the familiarization session (day 1 ), the rats did not receive any treatment and were allowed to explore the apparatus, which contained a neutral cloth. In the conditioning session (day 2), the rats received saline, propranolol, or nadolol and, 30 min later, were placed in the apparatus with the cat odor cloth. In the context session (day 3), rats received saline and were reexposed to the context session containing a neutral cloth.

Experiment 3: PMd-Fos immunoreactivity study in the groups treated with propranolol, nadolol, or saline before cat odor exposure. Subjects were randomly divided to receive propranolol $(10 \mathrm{mg} / \mathrm{kg}$, i.p.; $n=5)$, nadolol $(10 \mathrm{mg} / \mathrm{kg}$, i.p.; $n=5)$, or saline $(n=5), 30 \mathrm{~min}$ before a $10 \mathrm{~min}$ cat odor exposure.

Ninety minutes after cat odor exposure, each animal was deeply anesthetized with chloral hydrate $(150 \mathrm{mg} / \mathrm{kg})$ and perfused transcardially with a solution of $4.0 \%$ paraformaldehyde in $0.1 \mathrm{~m}$ phosphate buffer at $\mathrm{pH} 7.4$; the brain was removed and left overnight in a solution of $20 \%$ sucrose in $0.1 \mathrm{M}$ phosphate buffer at $4^{\circ} \mathrm{C}$. The brains were then frozen, and four series of $30 \mu \mathrm{m}$ sections were cut with a sliding microtome in the frontal plane and collected at the levels of the PMd. One complete series of sections was processed for immunohistochemistry with anti-Fos serum raised in rabbit (Ab-5, lot D09803; Oncogene Science) at a dilution of $1: 10,000$. The primary antiserum was localized using a variation of the avidin-biotin complex system (ABC). In brief, sections were incubated for $90 \mathrm{~min}$ at room temperature in a solution of biotinylated goat antirabbit IgG (Vector Laboratories) and then placed in the mixed avidinbiotin-horseradish peroxidase complex solution (ABC Elite kit; Vector Laboratories) for the same period of time. The peroxidase complex was visualized by a $10 \mathrm{~min}$ exposure to a chromogen solution containing $0.02 \% 3,3^{\prime}$ diaminobenzidine tetrahydrochloride with $0.3 \%$ nickel-ammonium sulfate (DAB-Ni) in $0.05 \mathrm{~m}$ Tris buffer, $\mathrm{pH}$ 7.6, followed by incubation for $10 \mathrm{~min}$ in chromogen solution with hydrogen peroxide (1:3000) to produce a blue-black product. The reaction was stopped by extensive washing in potassium-PBS, pH 7.4. Sections were mounted on gelatin-coated slides and then dehydrated and coverslipped with DPX.

Counts of the number of Fos-immunoreactive neurons as a function of experimental status were generated for the PMd by using the $10 \times$ 
objective of a Nikon Eclipse E600 microscope equipped with a camera lucida. To be considered as positive for Fos-like immunoreactivity, the nucleus of the neurons had to be of appropriate size (ranging approximately from 8 to 15 $\mu \mathrm{m}$ ) and shape (oval or round), show the characteristic blue-black staining of oxidized DAB$\mathrm{Ni}$, and be distinct from the background at magnification of $10 \times$. For each animal, Fospositive cells were plotted and counted at three distinct rostrocaudal levels of the PMd (120 $\mu \mathrm{m}$ apart).

Experiment 4: effects of atenolol microinjection in the PMd of rats exposed to cat odor or context environment. Rats were implanted with bilateral cannulas aimed at the PMd, randomly grouped to receive microinjections of $10 \mathrm{nmol}$ atenolol, $40 \mathrm{nmol}$ atenolol, or PBS $10 \mathrm{~min}$ before conditioning and were tested during cat odor exposure and, on the following day, during exposure to the cat odor-associated context. Another group of animals received microinjections of 40 $\mathrm{nmol}$ atenolol or PBS $10 \mathrm{~min}$ before exposure to the cat odor-associated context environment and were reexposed, $24 \mathrm{~h}$ later, to the same context environment (context II), to evaluate contextual conditioned behavioral responses in the absence of possible drug effects.

To evaluate the injection site placement, rats were deeply anesthetized with chloral hydrate $(150 \mathrm{mg} / \mathrm{kg})$ and were perfused transcardially with saline $(0.9 \% \mathrm{NaCl})$ and paraformaldehyde $(10 \%)$ for 10 min each. A volume of $0.2 \mu \mathrm{l}$ of Evans blue dye $(30 \mathrm{mg} / \mathrm{ml})$ was then administered through the same needle previously used in the experiments. After decapitation, the brain was removed from the skull and stored in 4\% paraformaldehyde solution ( $\mathrm{w} / \mathrm{v})$. At least $24 \mathrm{~h}$ before sectioning, the brains were immersed in a $30 \%$ sucrose solution $(\mathrm{w} / \mathrm{v})$ for cryoprotection. Coronal slices $(50 \mu \mathrm{m})$ were cut on a cryostat (Leica CM1850) and mounted on gelatin-coated slides. The sections were examined in an optical microscope to determine the injection sites delimited by the Evans blue dye.

Data analysis

Student's $t$ test for dependent samples was used to compare changes in the heart rate before and after $\beta$-blockers injections. ANOVA followed by Newman-Keuls test was used to compare the effects of the treatments on the mean percentage of approach time and hide time, the head-out time, and the number of PMdFos-positive cells. The level of 0.05 was considered to be statistically significant.

\section{Results}

Experiment 1: effects of propranolol and nadolol on the heart rate increase produced by dopamine systemic injection

As illustrated in Figure 1, both $\beta$-blockers, injected $30 \mathrm{~min}$ before, were able to prevent $(p<0.05)$ the tachycardia induced by different doses of dopamine $(18,36$, and $72 \mu \mathrm{g} / \mathrm{kg})$, without promoting alterations in the baseline heart rate. This experiment indicated that $10 \mathrm{mg} / \mathrm{kg}$ dose and $30 \mathrm{~min}$ injection interval time were adequate regimens for the use of the $\beta$-blockers in the behavioral experiments.

\section{Experiment 2: effects of propranolol and nadolol on rats' defensive behavior elicited in a cat odor apparatus}

On the familiarization day, no statistical difference was detected by ANOVA among the three groups for the behavior parameters observed in the apparatus. In general, rats spent $\sim 30 \%$ of the time near the neutral cloth and $\sim 30 \%$ of the time in the hide box (Fig. 2).

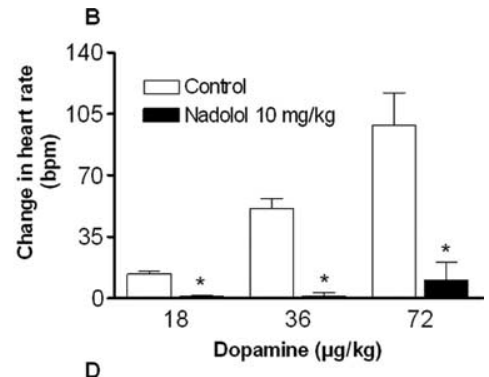

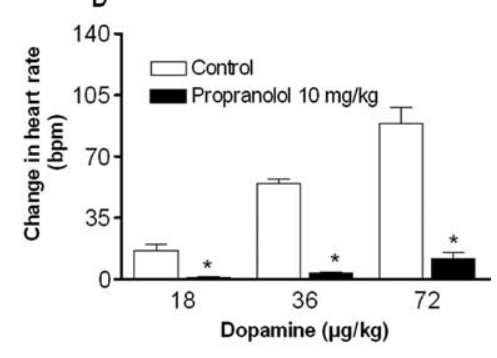
increases after dopamine $(18,36,72 \mu \mathrm{g} / \mathrm{kg})$ in anesthetized rats. $\boldsymbol{B}$ and
the groups $(n=3) .{ }^{*} p<0.05$ compared with the respective control.
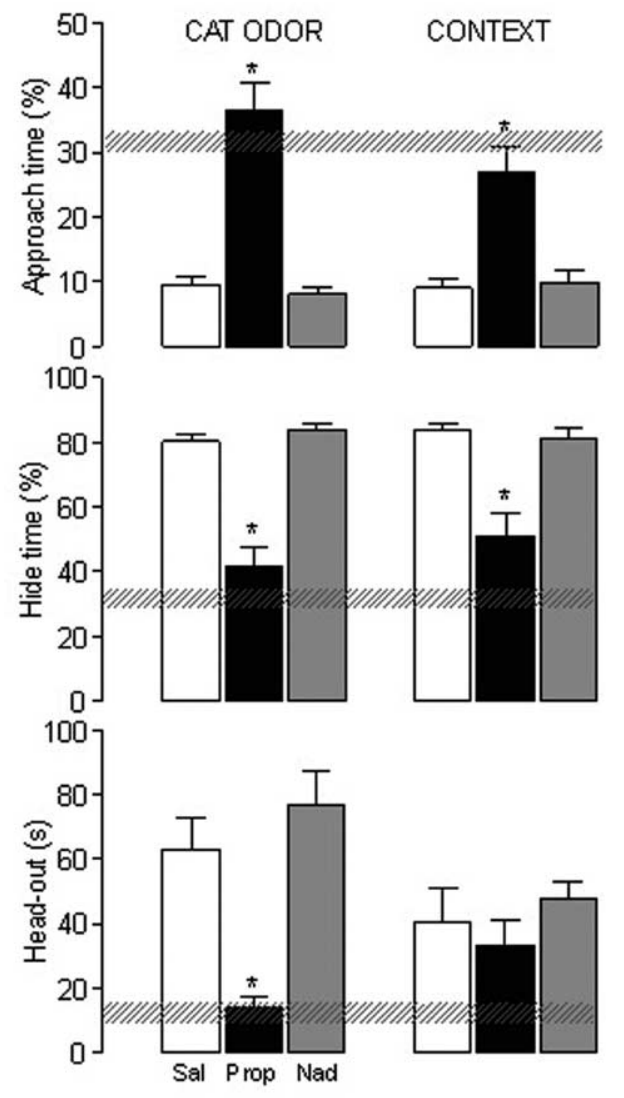

Figure 2. Experiment 2. The effects of propranolol (Prop; $10 \mathrm{mg} / \mathrm{kg}$, i.p.) and nadolol (Nad; $10 \mathrm{mg} / \mathrm{kg}$, i.p.) in the percentage of approach time or hide time and the head-out time in rats confronted to cat odor. The familiarization, conditioning, and context sessions were conducted during 3 consecutive days. Hatched horizontal bars represent the mean and the confidence limits ( $\pm 95 \%)$ for the familiarization data. Subjects received propranolol $(n=18)$, nadolol ( $n=18$ ), or saline (Sal; $n=27$ ) injections 30 min before the conditioning session. In the context session, the subjects received saline and were reexposed to the cat odor-associated context. Histograms represent the conditioning and context data and were expressed as mean + SEM. ${ }^{*} p<0.05$ (Newman-Keuls post hoc test) versus saline control group. 


\section{A Immunohistochemistry}
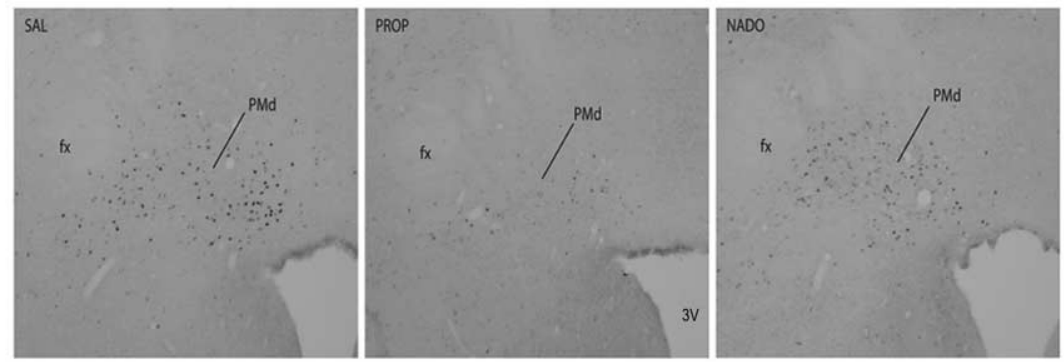

\section{B}

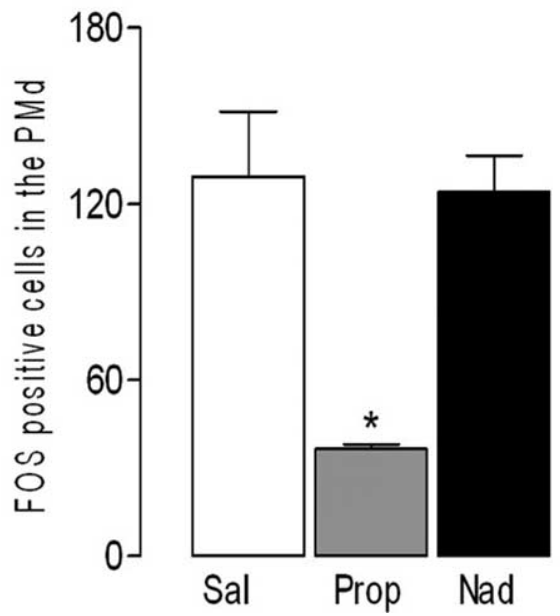

Figure 3. Experiment 3. Effects on the PMd-Fos immunoreactivity of saline (Sal), propranolol (Prop; $10 \mathrm{mg} / \mathrm{kg}$, i.p.), and nadolol (Nado; $10 \mathrm{mg} / \mathrm{kg}$, i.p.) injections $30 \mathrm{~min}$ before cat odor exposure. $\boldsymbol{A}$, Bright-field photomicrographs illustrating the PMd-Fos-positive cells in the saline (Sal)-, propranolol (Prop)-, and nadolol (Nad)-injected groups. fx, fornix; 3V, third ventricle. $\boldsymbol{B}$, Histogram showing the mean + SEM number of PMd-Fos-positive cells in the Sal-, Prop-, and Nad-treated groups. Data represent means + SEM for five rats per group. ${ }^{*} p<0.05$ compared with saline group. One-way ANOVA followed by NewmanKeuls test.

On the conditioning day, an ANOVA overall comparison showed a significant $(p<0.01)$ treatment effect among the groups for the percentage of approach time $\left(F_{(2,27)}=15.81\right)$, percentage of hide time $\left(F_{(2,27)}=17.79\right)$, and head-out time $\left(F_{(2,27)}=4.02\right)$. Post hoc comparisons revealed a significant $(p<0.05)$ effect of propranolol, but not of nadolol, in the above parameters when compared with the saline group. Propranolol-treated rats showed an increased time spent near the cat odor source and a decreased time in the hide box. In addition, the decreased head-out time after propranolol suggested a reduced risk assessment behavior (Fig. 2). No statistical difference was detected between saline- and nadololtreated subjects.

On the context day, ANOVA overall comparison continued to show a significant $(p<0.01)$ treatment effect among the groups for the percentage of approach time $\left(F_{(2,27)}=13.73\right)$ and percentage of hide time $\left(F_{(2,27)}=13.52\right)$. Post hoc comparisons revealed a significant $(p<0.05)$ effect in the subjects from the propranolol when compared with the saline group (Fig. 2). Therefore, the subjects treated with propranolol before the conditioning session displayed a significant $(p<0.05)$ reduction in the contextual conditioned fear. Finally, rats treated with nadolol or saline before the conditioning day failed to show reduction in the defensive behavior during the context day (Fig. 2).
Experiment 3: PMd-Fos

immunoreactivity study in the groups treated with propranolol, nadolol, or saline before cat odor exposure

ANOVA revealed statistical differences $\left(F_{(2,12)}=12.58 ; p<0.001\right)$ in the PMdFos-positive cells among the groups treated with propranolol, nadolol, or saline. Newman-Keuls post hoc comparisons revealed a decrease $(p<0.05)$ in the number of Fos-positive cells in the PMd of rats that had received propranolol $30 \mathrm{~min}$ before cat odor exposure (Fig. 3).

\section{Experiment 4: effects of atenolol} microinjection in the PMd of rats exposed to cat odor or to context Experiment 4.1: atenolol microinjection before cat odor exposure

Histological analysis revealed that nine animals from the PBS group and 14 animals that had received atenolol (seven injected with $10 \mathrm{nmol}$ and seven injected with 40 $\mathrm{nmol}$ ) had the injection placed in the PMd region. In another 15 animals that had received atenolol, the injection site hit either caudally (the medial mammillar nucleus, $n=10$ ) or rostrally (the ventromedial hypothalamic nucleus, $n=5$ ) to the region of the PMd, the A-out group.

No statistical differences among the groups were detected by ANOVA in the behavioral parameters evaluated during familiarization phase (Fig. 4). On the conditioning day, ANOVA showed a significant $(p<0.001)$ treatment effect in the defensive behavior, represented by the percentage of hide time $\left(F_{(3,34)}=14.23\right)$ and approach time $\left(F_{(3,34)}=9.29\right)$ and in the risk assessment behavior, measured by head-out time $\left(F_{(3,34)}\right.$ $=7.45)$. Newman-Keuls post hoc comparisons revealed a significant $(p<0.05)$ increase in the percentage of approach time and a decrease in the percentage of hide time and the head-out time in the groups microinjected with 10 or $40 \mathrm{nmol}$ atenolol into the PMd before cat odor exposure when compared with PBS and A-out groups (Fig. 4).

In the context session, $24 \mathrm{~h}$ later, ANOVA showed statistical differences $(p<0.001)$ among the groups in the percentage of approach time $\left(F_{(3,34)}=9.29\right)$, in the percentage of hide time $\left(F_{(3,34)}=14.23\right)$ and head-out time $\left(F_{(3,34)}=7.45\right)$. Post hoc comparisons detected a significant $(p<0.05)$ increase in the percentage of approach time and a reduction in the percentage of hide time in both groups microinjected with atenolol into the PMd before conditioning (Fig. 4). In addition, a decrease in the head-out time during context was visualized in the group microinjected with atenolol before cat odor exposure when compared with control group (Fig. 4).

Experiment 4.2: atenolol microinjection before context I

Histological analysis revealed that six animals from the PBS group and seven animals that had received atenolol had the injection placed in the PMd region. In another six animals that had received atenolol, the injection site hit either caudally (the medial 

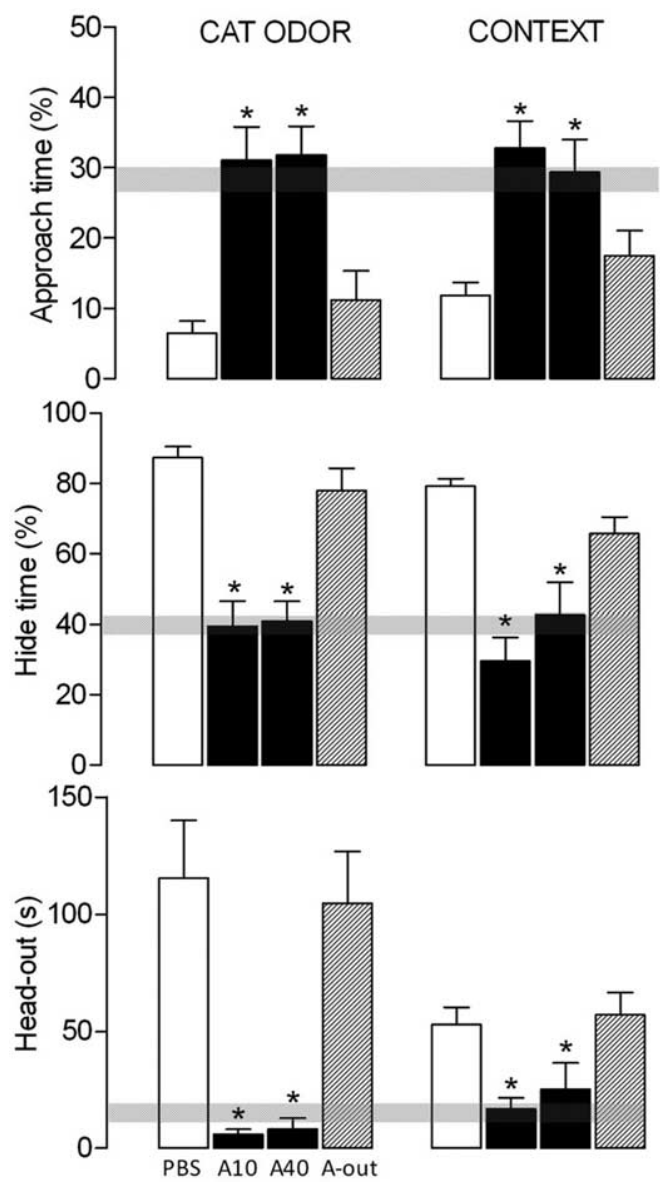

Figure 4. Experiment 4.1. Effects of atenolol, applied $10 \mathrm{~min}$ before the conditioning session on defensive behavior exhibited by rats exposed to a cat odor. The familiarization, the conditioning to the cat odor, and the context sessions were conducted during 3 consecutive days, during which the percentage of approach time, the percentage of hide time, and the head-out time were recorded in 10 min sessions each. Hatched horizontal bars represent the mean and the confidence limits $( \pm 95 \%)$ for the data obtained during the familiarization session. PBS $(n=9), 10 \mathrm{nmol}$ atenolol (A10; $n=7)$, and $40 \mathrm{nmol}$ atenolol (A40; $n=7)$ in the PMd and the A-out group $(n=15)$ were microinjected $(0.2 \mu l) 10$ min before the conditioning to the cat odor. ${ }^{*} p<0.05$ compared with PBS group.

mammillar nucleus, $n=5$ ) or rostrally (the ventromedial hypothalamic nucleus, $n=1$ ) to the region of the PMd, the A-out group.

No statistical differences among the groups were detected by ANOVA in the behavioral parameters evaluated during familiarization and conditioning sessions (Fig. 5).

On the context day (context I), ANOVA revealed statistical differences $(p<0.05)$ among the groups in the parameters headout time $\left(F_{(2,16)}=10.49\right)$ and the percentage of hide time $\left(F_{(2,16)}\right.$ $=5.49)$. Newman-Keuls post hoc comparison revealed that subjects microinjected with atenolol into the PMd 10 min before context I showed a reduced $(p<0.05)$ percentage of hide time and head-out time behaviors (Fig. 5). No significant effects were observed $24 \mathrm{~h}$ later during the second exposition to the same context (context II) when compared with PBS and A-out groups (Fig. 5).

\section{Discussion}

The set of results presented here support the hypothesis that defensive behavior exhibited by rats in response to cat odor stimulus are under the influence of $\beta$-adrenergic neurotransmission.
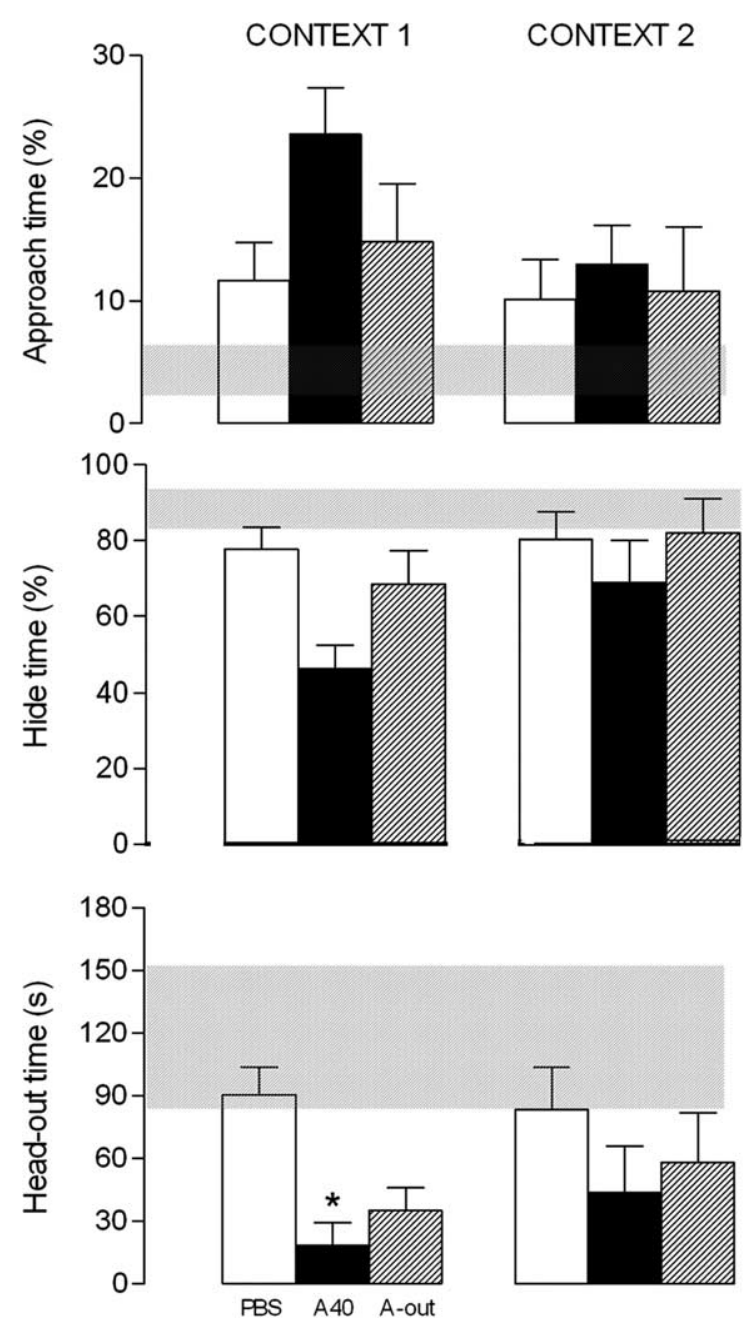

Figure 5. Experiment 4.2. Effects of atenolol, applied $10 \mathrm{~min}$ before the first context session, on defensive behavior exhibited by rats exposed to a cat odor. The familiarization, the conditioning to the cat odor, and the two context sessions were conducted during 4 consecutive days, during which the percentage of approach time, the percentage of hide time, and the head-out time were recorded in 10 min sessions each. Hatched horizontal bars represent the mean and the confidence limits $(p<0.05)$ for the data obtained during the conditioning session. Percentage of approach time, percentage of hide time, and head-out time in the groups microinjected with PBS $(n=6)$ and $40 \mathrm{nmol}$ atenolol $(\mathrm{A} 40 ; n=7)$ in the PMd and the A-out group $(n=6) 10$ min before the first context exposure. ${ }^{*} p<0.05$ compared with control group.

Evidence has shown that the noradrenergic influence on these responses depends on a central, rather than a peripheral, $\beta$-adrenergic action, which seems to be primarily mediated by the PMd, the hypothalamic site most responsive to predatory threats (Canteras et al., 1997, 2008; Dielenberg et al., 2001; Cezario et al., 2008; Staples et al., 2008).

Considering that $\beta$-blockade-induced impaired cardiovascular function can lead to a possible reduction in the locomotor activity, we initially tested the appropriate propranolol and nadolol doses in anesthetized rats, which were infused with increasing doses of dopamine. Both $\beta$ antagonists, at the dose of $10 \mathrm{mg} / \mathrm{kg}$, were able to block the tachycardia induced by all doses of dopamine without effects on the heart rate baseline.

After defining the $\beta$-antagonist dose, rats were exposed to the cat odor apparatus to evaluate the effects of both $\beta$-blocker agents on the innate and learned defensive responses toward predator odor. Propranolol, given immediately before cat odor exposure (conditioning session), was able to reduce the defensive behavior 
when compared with the saline-treated group. Propranololtreated rats spent more time exploring the area near the cat odor source and less time hiding or performing risk assessment behavior. In sharp contrast, nadolol, a $\beta$-blocker that does not cross the blood-brain barrier (Cruickshank and Prichard, 1987), did not alter defensive responses during cat odor exposure, showing that central, rather than peripheral, $\beta$-adrenergic blockade is able to impair unconditioned responses to predator odor. Supporting the idea of a central noradrenergic neurotransmission role during exposure to predatory threats, previous studies reported that the locus ceruleus is particularly activated during exposure to a predator or to its odor (Dielenberg et al., 2001; Ribeiro-Barbosa et al., 2005). Together, these findings lead to the important conclusion that central noradrenergic mediation seems to be critical for the expression of innate fear responses to predatory threats.

Likewise, propranolol, but not nadolol, pretreatment before the conditioning session impaired the acquisition of the contextual conditioned fear. The effects of $\beta$ adrenoceptors on the consolidation processes of aversive memories have been consistently demonstrated in studies using shock-based inhibitory avoidance, as well as shock-based contextual and cued fear conditioning (McGaugh, 2004). However, in contrast to the present findings suggesting a central, and not a peripheral, $\beta$-adrenergic mediation on contextual conditioning to predator odor, the adrenergic influence on memory consolidation in shock-based procedures seems to depend on a peripheral neuronal pathway involving $\beta$ adrenoceptors on vagal afferents terminating on brainstem noradrenergic cell groups in the nucleus of the solitary tract (McGaugh and Roozendaal, 2002). These contrasting results between predator odor-based and shock-based procedures may reflect the different nature and processing of the two aversive stimuli, the former involving primarily psychological processing and the latter representing a physically aversive stimulus, which is likely to engage more interoceptive signaling.

We have further investigated the neural systems mediating the noradrenergic modulation of antipredatory defensive behavior and chosen the PMd to start addressing this matter. As mentioned previously, the PMd represents the hypothalamic site most responsive to predatory threats (Canteras et al., 1997, 2008; Dielenberg et al., 2001; Cezario et al., 2008; Staples et al., 2008) and, at the same time, presents a dense plexus of noradrenergic fibers and terminals (Moore and Card, 1984). In the present work, we were able to show that propranolol, but not nadolol, drastically reduced PMd-Fos expression in response to cat odor, thus suggesting a strong $\beta$-adrenergic modulation of the nucleus during exposure to predator odor.

At this point, it would be interesting to have a comprehensive analysis of the neural sites potentially influenced by the $\beta$-adrenergic blockade during predator odor exposure. This investigation would certainly help to provide an overall view on the $\beta$-adrenergic blockade impact on the neural systems processing predator threats. However, the emerging picture of this investigation would be difficult to interpret, especially considering that the drastic decrease in the PMd activation (induced by $\beta$-adrenergic blockade) by itself is likely to influence the activation pattern of key regions responding to predatory threats (Cezario et al., 2008).

To test whether the PMd would be involved in mediating the noradrenergic influence in innate and learned defensive responses to predator odor, local injections of $\beta$-blocker were applied in the PMd before cat odor exposure. The use of propranolol in this experiment seems to have a potential drawback, because nonselective $\beta$ antagonists may potentially have seroto- nin (5-HT) antagonist properties that could interfere with antidefensive effects (Costain and Green, 1978). However, based on the literature, it is unlikely that the propranolol antidefensive effects would be mediated by 5-HT, especially considering that a number of 5-HT antagonists failed to show any behavioral effect in cat odor scenario (Shepherd et al., 1992, 1993; Dielenberg and McGregor, 2001). Despite the evidence, to avoid any possible blockade of 5-HT receptors, we tested the selective $\beta$ 1-blocker atenolol for local injections within the PMd. These injections were made immediately before the exposure to cat odor or cat odor-related environment. The results showed that the $\beta$-adrenoceptor blockade in the PMd, but not in the adjacent sites, 10 min before the conditioning session, reduced the defensive responses to the cat odor and also, $24 \mathrm{~h}$ later, to the cat odor-related environment. Therefore, these results suggest that the PMd is a critical site mediating $\beta$-adrenergic modulation of the unconditioned defensive behavior, seemingly influencing the acquisition of the contextual fear conditioning to cat odor.

The functional roles of the PMd appear to depend on its branched pathway to the periaqueductal gray (PAG) and to the ventral part of the anteromedial thalamic nucleus (Canteras and Swanson, 1992; Cezario et al., 2008). Whereas the PAG appears to influence antipredatory defensive responses (Cezario et al., 2008), the ventral part of the anteromedial thalamic nucleus projects to the lateral retrosplenial area and is thought to be involved in modulating the eye and head movements associated with attentional processes (Risold and Swanson, 1995). In fact, we have shown recently that the PMd influences the activation of its major projecting targets in response to predatory threats (i.e., the PAG and the ventral part of the anteromedial thalamic nucleus) but does not seem to affect the amygdalar sites involved in predator's cue detection (Cezario et al., 2008).

The PMd $\beta$-blockade-induced deficit in contextual fear conditioning to cat odor may be viewed as either an impairment in the memory consolidation process or the result of the decreased emotional component of the aversive event during the learning stage. To date, we have no experimental evidence showing PMd targets as involved in aversive memory processing; therefore, it is more parsimonious to believe that the decrease in the emotional responses during cat odor exposure would be accountable for the deficit in the acquisition of the contextual fear conditioning. In line with this view, recent findings from our laboratories have also shown that, during cat odor exposure, NMDA receptor antagonist injections in the PMd were also able to block contextual fear conditioning (Canteras et al., 2008). We also shown that atenolol, microinjected in the PMd only before the context session, reduced the expression of the defensive response acquired during the conditioning session $24 \mathrm{~h}$ earlier. Therefore, the PMd also appears to mediate a $\beta$-adrenoceptor modulation on the expression of contextual conditioned responses to cat odor. In line with this view, pharmacological inactivation of the PMd, but not of the nearby mammillary nuclei, was able to practically abolish the contextual conditioned responses to predatory threats, an effect that seems to be mediated through the projections to the periaqueductal gray (Cezario et al., 2008). Here it is noteworthy that exposure to a predatory context upregulates Fos expression in the locus ceruleus (Ribeiro-Barbosa et al., 2005; Staples et al., 2008). Thus, similarly to what was discussed for antipredatory unconditioned responses, the findings support the idea that central noradrenergic mediation also seems to be critical for the expression of learned fear responses to predatory threats.

The possibility that a $\beta$-adrenoceptor blockade in the PMd, during exposure to cat odor-associated context $24 \mathrm{~h}$ after expo- 
sure to cat odor, would elicit a carryover effect in subsequent sessions was ruled out by the marked defensive behavior expressed by the same subjects when reexposed to the same context $24 \mathrm{~h}$ later (context II) in the absence of any drug effect.

Overall, the present results provide convincing evidence suggesting a central, but not peripheral, $\beta$-adrenoceptor mediation of defensive behavior to cat odor, influencing both unconditioned and conditioned responses. We have further shown that the PMd appears to be a critical locus to mediate all these $\beta$-adrenoceptor effects.

\section{References}

Angrini M, Leslie JC, Shephard RA (1998) Effects of propranolol, buspirone, pCPA, reserpine, and chlordiazepoxide on open-field behavior. Pharmacol Biochem Behav 59:387-397.

Apfelbach R, Blanchard CD, Blanchard RJ, Hayes RA, McGregor IS (2005) The effects of predator odors in mammalian prey species: a review of field and laboratory studies. Neurosci Biobehav Rev 29:1123-1144.

Audi EA, de Oliveira RM, Graeff FG (1991) Microinjection of propranolol into the dorsal periaqueductal gray causes an anxiolytic effect in the elevated plus-maze antagonized by ritanserin. Psychopharmacology 105:553-557.

Bremner JD, Krystal JH, Southwick SM, Charney DS (1996) Noradrenergic mechanisms in stress and anxiety. I. Preclinical studies. Synapse 23:28-38.

Brunello N, den Boer JA, Judd LL, Kasper S, Kelsey JE, Lader M, Lecrubier Y, Lepine JP, Lydiard RB, Mendlewicz J, Montgomery SA, Racagni G, Stein MB, Wittchen HU (2000) Social phobia: diagnosis and epidemiology, neurobiology and pharmacology, comorbidity and treatment. J Affect Disord 60:61-74.

Canteras NS, Swanson LW (1992) The dorsal premammillary nucleus: an unusual component of the mammilary body. Proc Natl Acad Sci U S A 89:10089-10093.

Canteras NS, Chiavegatto S, Valle LE, Swanson LW (1997) Severe reduction of rat defensive behavior to a predator by discrete hypothalamic chemical lesions. Brain Res Bull 44:297-305.

Canteras NS, Kroon JA, Do-Monte FH, Pavesi E, Carobrez AP (2008) Sensing danger through the olfactory system: The role of the hypothalamic dorsal premammillary nucleus. Neurosci Biobehav Rev 32:1228-1235.

Cezario AF, Ribeiro-Barbosa ER, Baldo MV, Canteras NS (2008) Hypothalamic sites responding to predator threats: the role of the dorsal premammillary nucleus in unconditioned and conditioned antipredatory defensive behavior. Eur J Neurosci 28:1003-1015.

Costain DW, Green AR (1978) Beta-adrenoceptor antagonists inhibit the behavioral responses of rats to increased brain 5-hydroxytryptamine. Br J Pharmacol 64:193-200.

Cruickshank JM, Prichard BNC (1987) Betablockers in clinical practice. Edinburgh: Churchill Livingstone.

Davidson JR (2006) Pharmacotherapy of social anxiety disorder: what does the evidence tell us? J Clin Psychiatry 67 [Suppl 12]:20-26.

Dielenberg RA, McGregor IS (2001) Defensive behavior in rats towards predatory odors: a review. Neurosci Biobehav Rev 25:597-609.

Dielenberg RA, Arnold JC, McGregor IS (1999) Low-dose midazolam at- tenuates predatory odor avoidance in rats. Pharmacol Biochem Behav 62:197-201.

Dielenberg RA, Hunt GE, McGregor IS (2001) When a rat smells a cat: the distribution of Fos immunoreactivity in rat brain following exposure to a predatory odor. Neuroscience 104:1085-1097.

Fuster JM (1997) Network memory. Trends Neurosci 20:451-459.

Giles J (2005) Beta-blockers tackle memories of horror. Nature 436:448-449.

Gorman AL, Dunn AJ (1993) Beta-adrenergic receptors are involved in stress-related behavioral changes. Pharmacol Biochem Behav 45:1-7.

Hirschmann S, Dannon PN, Iancu I, Dolberg OT, Zohar J, Grunhaus L (2000) Pindolol augmentation in patients with treatment-resistant panic disorder: a double-blind, placebo-controlled trial. J Clin Psychopharmacol 20:556-559.

Kent JM, Mathew SJ, Gorman JM (2002) Molecular targets in the treatment of anxiety. Biol Psychiatry 52:1008-1030.

McGaugh JL (2004) The amygdala modulates the consolidation of memories of emotionally arousing experiences. Annu Rev Neurosci 27:1-28.

McGaugh JL, Roozendaal B (2002) Role of adrenal stress hormones in forming lasting memories in the brain. Curr Opin Neurobiol 12:205-210.

Moore RY, Card JP (1984) Noradrenaline-containing neuron systems. In: Handbook of chemical neuroanatomy, Vol 2, Classical transmitters in the CNS, Part 1 (Björklund A, Hökfelt T, eds), pp 123-156. Amsterdam: Elsevier.

Morilak DA, Barrera G, Echevarria DJ, Garcia AS, Hernandez A, Ma S, Petre CO (2005) Role of brain norepinephrine in the behavioral response to stress. Prog Neuropsychopharmacol Biol Psychiatry 29:1214-1224.

Paxinos G, Watson C (1997) The rat brain in stereotaxic coordinates, Ed 3. Sydney: Academic.

Ribeiro-Barbosa ER, Canteras NS, Cezário AF, Blanchard RJ, Blanchard DC (2005) Na alternative experimental procedure for studying predatorrelated defensive responses. Neurosci Biobehav Rev 29:1255-1263.

Risold PY, Swanson LW (1995) Evidence for a hypothalamocortical circuit mediating pheromonal influences on eye and head movements. Proc Natl Acad Sci U S A 92:3898-3902.

Shepherd JK, Flores T, Rodgers RJ, Blanchard RJ, Blanchard DC (1992) The anxiety/defense test battery: influence of gender and ritanserin treatment on antipredator defensive behavior. Physiol Behav 51:277-285.

Shepherd JK, Rodgers RJ, Blanchard RJ, Magee LK, Blanchard DC (1993) Ondansetron, gender and antipredator defensive behaviour. J Psychopharmacol 7:72-81.

Staples LG, McGregor IS, Apfelbach R, Hunt GE (2008) Cat odor, but not trimethylthiazoline (fox odor), activates accessory olfactory and defenserelated regions in rats. Neuroscience 151:937-947.

Stern CA, Carobrez AP, Bertoglio LJ (2008) Aversive learning as a mechanism for lack of repeated anxiolytic-like effect in the elevated plus-maze. Pharmacol Biochem Behav 90:545-550.

Sullivan GM, Coplan JD, Kent JM, Gorman JM (1999) The noradrenergic system in pathological anxiety: a focus on panic with relevance to generalized anxiety and phobias. Biol Psychiatry 46:1205-1218.

Vaiva G, Ducrocq F, Jezequel K, Averland B, Lestavel P, Brunet A, Marmar CR (2003) Immediate treatment with propranolol decreases posttraumatic stress disorder two months after trauma. Biol Psychiatry 54:947-949. 